\title{
RELAÇÃO ENTRE PARÂMETROS ANTROPOMÉTRICOS, BIOQUÍMICOS E ESTILO DE VIDA DE INDIVÍDUOS ATENDIDOS EM UM AMBULATÓRIO DE SAÚDE
}

\section{RELATION AMONG ANTHROPOMETRIC PARAMETERS, BIOCHEMICALS AND LIFESTYLE OF INDIVIDUALS IN A HEALTH AMBULATORY}

\author{
Juliana Diniz Ribeiro* (D), Bianca Coletti Schauren $(\mathbb{D}$ \\ Universidade do Vale do Taquari, Lajeado, RS, Brasil. \\ *julianadinizr@gmail.com
}

\section{RESUMO}

Apesar do maior acesso a informações e conscientização sobre prevenção de doenças e promoção da saúde, a situação de saúde atual da população brasileira ainda é preocupante. Neste sentido, este estudo teve por finalidade avaliar a relação entre os parâmetros antropométricos, bioquímicos e estilo de vida de indivíduos acompanhados em um ambulatório de saúde na região central do estado do RS. Trata-se de um estudo transversal, quantitativo, descritivo, realizado com adultos e idosos no ano de 2018. Foi avaliado o estado nutricional por meio de parâmetros antropométricos, bioquímicos e estilo de vida. Participaram do estudo 57 indivíduos, com idade média de 40,6 $\pm 14,6$ anos e 53,8\% mulheres. Quanto ao motivo da consulta, indivíduos obesos intencionavam atingir a redução de peso e indivíduos eutróficos à reeducação alimentar. Verificou-se diferença significativa no aumento dos níveis da glicose de jejum (pré-diabetes) nos obesos. Aumento do risco cardiovascular segundo a circunferência abdominal e relação cintura/estatura em indivíduos com sobrepeso e obesidade. Já a circunferência do braço apresentou-se alterada nos indivíduos eutróficos (depleção muscular) e nos obesos (excesso de gordura). Os homens apresentaram valores significativamente inferiores da lipoproteína de alta densidade. Já as mulheres apresentaram associação significativa com risco cardiovascular aumentado. Com este estudo, observou-se nesta população maior risco cardiovascular e dos marcadores bioquímicos em indivíduos com sobrepeso e obesidade. Estes achados, reforçam a relevância de atuar na mudança de estilo de vida e no perfil de saúde para prevenção e tratamento de complicações metabólicas em adultos e idosos.

Palavras-chave: Doenças crônicas não transmissíveis. Estado nutricional. Risco cardiovascular.

\section{ABSTRACT}

Despite greater access information and awareness about illness prevention and health promotion, the current Brazilian population health still is worrying. In this sense, this study has the purpose of evaluating the relations among anthropometric parameters, biochemical and lifestyle of individuals followed in a health outpatient from the central region of the RS state. It is a transversal, quantitative and descriptive study, conducted with adults and elderly patients in the year of 2018. The nutritional status was rated through anthropometric parameters, laboratory, and lifestyle. Fifty-seven individuals had participated in the study; the average age was 40.6 years $\pm 14,6$ and $53.8 \%$ were women. As for the reason for the appointment, obese individuals intended to achieve weight reduction and eutrophic individuals to achieve dietary reeducation. There was a significant difference in the increase of fasting glucose levels (pre-diabetes) in the obese. There was also an increase in cardiovascular risk according to waist circumference and waist/height ratio in overweight and obese individuals. The circumference of the arm, on the other hand, was different in eutrophic individuals (muscle depletion) and in obese individuals (excess fat). Men had significantly lower values of high-density lipoprotein. Women, on the other hand, had a significant association with increased cardiovascular risk. With this study, a higher cardiovascular risk and biochemical markers were observed in this population of overweight and obese individuals. These findings reinforce the relevance of acting on lifestyle changes and on health profile for the prevention and treatment of metabolic complications in adults and elderly.

Keywords: Cardiovascular risk. Non-transmissible chronic diseases. Nutritional status. 


\section{INTRODUÇÃO}

Nos últimos anos, a sociedade teve um crescente aumento no acesso a informações sobre prevenção de doenças e promoção da saúde, aumentando a busca por espaços que atuam com educação alimentar e nutricional (FRANGELLA; TCHAKMAKIAN; PEREIRA, 2007; BRASIL, 2012; CERVATO-MANCUSO, VINCHA; SANTIAGO, 2016; SILVA et al., 2018). Este incremento na demanda por suporte para a melhoria do perfil de saúde se deve a situação de saúde atual da população brasileira, representado por um perfil crescente de doenças crônicas aumentando a morbimortalidade tanto em adultos como idosos (BRASIL, 2014; BRASIL, 2019).

No Brasil, as Doenças Cardiovasculares (DCVs) representaram a quinta causa de mortalidade entre a faixa etária de 20 a 39 anos no ano de 2015. Já entre a faixa etária de 40 e 59 anos de idade, as DCVs isquêmicas constituíram a primeira causa de morte, para ambos os sexos, com taxa de mortalidade duas, três vezes maior nos homens. Embora as DCVs sejam a principal causa de morte no sexo feminino, o risco de morte por esta doença é 1,2 vezes maior nos homens, representando a segunda causa de morte. No sexo masculino prevalece a doença cardíaca isquêmica, com risco de morte 1,5 vezes maior que no grupo feminino (BRASIL, 2018).

Bem como as DCVs, Hipertensão Arterial Sistêmica (HAS), Diabetes Mellitus (DM) e obesidade encontram-se em destaque dentre as Doenças Crônicas não Transmissíveis (DCNTs). Estas patologias possuem uma severidade maior sabendo que dispõem da capacidade concomitante de a aumentar incidência uma da outra (CUPPARI, 2009; BRASIL, 2013; BRASIL, 2015). Entre os principais fatores de risco associados a desenvolvimento das DCNTs estão o estilo de vida sedentário e hábitos alimentares inadequados ligados ao excesso de peso (VIGITEL, 2018).

A prática de atividade física regular contribui para maior qualidade de vida, reduz o risco do desenvolvimento de DCNTs e também reduz o risco de mortalidade (CARLUCCI et al., 2013). Os indivíduos sedentários apresentam 20 a 30\% mais risco de mortalidade do que aqueles que praticam atividade física (WHO, 2010). Assim como, hábitos alimentares inadequados como o consumo de alimentos industrializados, levam a um risco potencial para o desenvolvimento de DCNTs (BRASIL, 2018). Segundo Moreira et al. (2015), os países de renda baixa e também de renda média como o Brasil, encaram o marketing bastante intenso da indústria em favor do consumo de alimentos ultraprocessados, levando a rápidas e negativas transições alimentares na população. Além disso, verificaram que a redução do consumo de alimentos processados e ultraprocessados na população do Reino Unido poderia resultar em 10 a 13\% (17.000 a 22.000) menos mortes por DCVs até o ano de 2030 e ainda mesmo sendo quase impossível eliminar o consumo destes alimentos da rotina alimentar desta população, esta redução certamente pode proporcionar benefícios importantes (MOREIRA et al., 2015). No Brasil, o consumo de alimentos processados está presente em $9,8 \%$ da população e de ultraprocessados em 18,4\%. Na região Sul do país, o consumo de alimentos processados representa $8,5 \%$ e de ultraprocessados em $22 \%$ (IBGE, 2020).

Dados do estudo sobre Vigilância de Fatores de Risco para DCNTs realizado no Brasil entre 2006 a 2017 com adultos ( $\geq 18$ anos) e idosos ( $\geq 65$ anos) identificou uma prevalência de $54 \%$ de excesso de peso, predominando nos homens e 13,9\% de inatividade física nos últimos 3 meses para ambos os sexos (VIGITEL, 2018). Segundo Amorim, Burgos e Cabral (2017), em idosos com DM a inatividade física esteve associada ao excesso de peso em indivíduos de ambos os sexos. Por isso, conhecer o perfil de saúde da população é de extrema importância para prevenção e redução da morbimortalidade.

Além disso para acompanhamento do perfil de saúde e/ou controle das DCNTs, destaca-se a importância da avaliação dos marcadores bioquímicos laboratoriais (BRASIL, 2018). Segundo a American Diabetes Association (ADA), glicemia de jejum alterada e tolerância a glicose diminuída estão relacionadas com o aumento do risco de DCVs. No caso do perfil lipídico, quanto mais altos forem os níveis de lipídeos e lipoproteínas de baixa densidade circulantes maior será o risco de DCVs (BRASIL, 2017). 
Diante deste contexto, este estudo teve por finalidade avaliar a relação entre parâmetros antropométricos, bioquímicos e de estilo de vida de indivíduos acompanhados em um ambulatório de saúde na região central do estado do Rio Grande do Sul (RS).

\section{MATERIAL E MÉTODOS}

Trata-se de um estudo transversal, quantitativo, descritivo, realizado em adultos e idosos em um Ambulatório de uma Empresa prestadora de serviços de saúde, situada na região central do RS, no período de julho a outubro de 2018. Este estudo teve a aprovação do Comitê de Ética em Pesquisa da Univates (COEP/UNIVATES) com o número de CAAE 91029318.2.0000.5310.

Participaram do estudo indivíduos de ambos os sexos, com idade $\geq$ a 20 anos que buscaram atendimento nutricional no ambulatório entre julho de 2018 a outubro de 2018 mediante a assinatura do Termo de Consentimento Livre e Esclarecido (TCLE). Foram excluídos do estudo oito pacientes por não apresentarem informações suficientes para a análise da pesquisa.

Foram avaliados o estado nutricional por meio de parâmetros antropométricos, exames bioquímicos e estilo de vida dos indivíduos. Adicionalmente, foram avaliadas doenças existentes, número de medicamentos utilizados, função intestinal pela escala de Bristol (1997), função urinária (cor, aspecto da urina) pela escala de Armstrong (1994) e exames bioquímicos realizados como glicemia jejum, colesterol total (CT) e frações (LDL, HDL, TG), além do motivo do acompanhamento nutricional. Adicionalmente foram coletas as variáveis de estilo de vida, prática de atividade física, tipo, duração, frequência e intensidade, avaliada pela escala de Borg (2000) e a atividade profissional atual. As variáveis foram coletadas utilizando-se um questionário estruturado com perguntas padronizadas.

O estado nutricional foi obtido por meio da aferição do peso em quilogramas $(\mathrm{kg})$ e altura em metros $(\mathrm{m})$ e circunferências $(\mathrm{cm})$. O peso foi aferido em uma balança mecânica da marca Cauduro $L d t a \AA$, com o indivíduo descalço e com o mínimo de roupas possível, a altura foi avaliada no estadiômetro da mesma balança com o indivíduo em pé, descalço, com calcanhares unidos, com glúteo, parte superior das costas e calcanhares em contato com o estadiômetro, braços ao lado do corpo e com a cabeça no plano de Frankfurt (STEWART et al., 2011). Por meio do cálculo, peso $(\mathrm{kg}) /$ altura $(\mathrm{m})^{2}$ foi obtido o Índice de Massa Corporal (IMC) e a classificação conforme o proposto pela Organização Mundial da Saúde (WHO/OMS) (1997) para os indivíduos adultos e de acordo com a Organização Pan-americana da Saúde (OPAS) (2002) para os indivíduos idosos. Para análise do estado nutricional e marcadores bioquímicos foi utilizada a análise parcial das amostras devido a disponibilidade destas informações.

As circunferências foram aferidas e classificadas de acordo com os padrões internacionais para avaliação antropométrica recomendados pela International Society for the Advancement of Kinanthropometry. A Circunferência Abdominal (CA) foi aferida e avaliada segundo a Organização Mundial da Saúde (OMS). Risco cardiovascular aumentado pode ser identificado quando a CA apresentada for $\geq 80 \mathrm{~cm}$ para mulheres e $\geq$ a $94 \mathrm{~cm}$ para homens e o risco muito aumentado quando a CA for $\geq 88 \mathrm{~cm}$ para mulheres e $\geq 102 \mathrm{~cm}$ para os homens (ABESO, 2009). Adicionalmente utilizou-se a Relação Cintura/Estatura (RCE) para avaliar risco cardiovascular, obtida pelo cálculo: cintura $\div$ altura $\mathrm{x} 100$. Valores $<0,5$ foram classificados como sem risco cardiovascular e valores $\geq$ a 0,5 foram classificados como risco cardiovascular (FONTES et al., 2018).

A Circunferência do braço $(\mathrm{CB})$ foi utilizada como parâmetro para avaliação do estado nutricional, foi obtida por meio do cálculo: adequação da $\mathrm{CB}(\%)=\mathrm{CB}$ obtida $(\mathrm{cm}) / \mathrm{CB}$ referência 50 x 100 e a classificação: depleção grave: $<70 \%$, depleção moderada: $70-80 \%$, depleção leve: 80 90\%, eutrofia: 90 - 110\%, sobrepeso: 110 - 120\%, obesidade: > 120\% (MUSSOI, 2014).

Os diagnósticos das doenças presentes nos indivíduos avaliados foram realizados previamente pelo médico assistente e coletadas em prontuário. A análise estatística foi realizada no programa Statistical Package for Social Science (SPSS) versão 22.0 por meio de estatística descritiva e testes 
de associação Exato de Fischer, teste não-paramétrico Kruskal-Wallis e teste não-paramétrico MannWhitney. Os dados foram considerados estatisticamente significativos quando o $\mathrm{p}<0,05$.

\section{RESULTADOS E DISCUSSÃO}

Participaram do estudo 57 indivíduos com idade média de 40,6 \pm 14,6 anos (mínimo: 20; máximo: 80) sendo a maioria adultos e do sexo feminino. No estudo de Saccon et al. (2015), foram avaliados 785 pacientes, sendo que $73,7 \%(n=578)$ eram do sexo feminino e variavam a idade entre 46,4 $\pm 15,4$ anos. Já no estudo de Amorim, Burgos e Cabral (2017), foram avaliados um total de 244 indivíduos idosos, com idade média de $69,7 \pm 7$ anos, sendo $82,4 \%$ mulheres. Foi possível observar que em ambas as pesquisas houve predominância do sexo feminino entre os participantes, assim como neste estudo. Este fato pode ser explicado pelo motivo de que as mulheres estão em maior número na população brasileira (51,7\%) enquanto homens 48,3\% (IBGE, 2018), além de acessarem os serviços de saúde com mais frequência (MOREIRAS, 2015).

No que se refere ao estado civil, $49,1 \%(n=28)$ eram solteiros e $47,4 \%(n=27)$ casados. Corroborando com o estudo, Santos et al. (2018), avaliaram 89 mulheres adultas, sendo a maioria, casadas (46\%). Já Reis e Almeida (2018), evidenciaram em seu estudo que 51,6\% $(\mathrm{n}=33)$ eram solteiros, $42,2 \%(\mathrm{n}=27)$ casados e $6,3 \%(\mathrm{n}=4)$ divorciados/viúvos. Os resultados de ambas as pesquisas podem ser explicados conforme a idade dos indivíduos participantes de cada pesquisa. A média de idade dos indivíduos colabora com estado civil atual.

Em relação as patologias existentes conforme diagnóstico médico prévio, $38,6 \%(n=22)$ não apresentavam nenhuma patologia, 19,3\% $(\mathrm{n}=11)$ apresentavam o diagnóstico de Diabetes Mellitus $(\mathrm{DM})$ ou pré-diabetes e $12,3 \%(\mathrm{n}=7)$ com diagnóstico de hipertensão arterial (HAS). Em discordância com o estudo, Santos et al. (2018) observaram que entre as patologias referidas mais prevalentes estavam, 37\% dos indivíduos com HAS, seguido de dislipidemia (24,8\%), DM (19\%), DCVs $(1,1 \%)$ e ainda, $47,1 \%$ dos indivíduos relataram apresentar outras patologias, as quais incluíam hipotireoidismo, esteatose hepática e depressão. Assim como no estudo de Saraiva, Zemolin, Zanardo (2014), a HAS foi a patologia mais prevalente $(21,49 \%)$, mas tal como neste estudo a maior parte $(41,12 \%)$ não apresentaram nenhuma patologia. As duas patologias presentes em maior parte neste estudo, e, como visto também encontradas nos estudos citados acima, HAS e DM apresentaram maior frequência devido ao fato de serem as patologias crônicas mais prevalentes na população brasileira (MALTA et al., 2015; STOPA et al., 2018).

Quanto ao motivo do acompanhamento $63,2 \%(\mathrm{n}=36)$ buscaram o serviço para redução do peso e $15,8 \%(\mathrm{n}=9)$ para reeducação alimentar. Assim como, no estudo Reis e Almeida (2018), evidenciaram que o principal objetivo do acompanhamento nutricional foi para redução do peso $(52,5 \%)$, seguido de reeducação alimentar $(36,1 \%)$ e, por fim, tratamento de patologias $(11,5 \%)$. Já, no estudo de Carvalho et al. (2015), que apesar de redução do peso (55,3\%) também estar como maior busca do atendimento nutricional, verificaram que $39,1 \%$ dos participantes buscaram o acompanhamento para o tratamento para patologias. Estes achados evidenciam uma maior preocupação e busca por espaços de promoção da educação alimentar e nutricional em benefício da saúde e qualidade de vida.

De acordo com a classificação do IMC, 38,6\% $(\mathrm{n}=22)$ tinham excesso de peso, respectivamente e $22,8 \%(\mathrm{n}=13)$ eram eutróficos. Observa-se que o motivo do acompanhamento esteve significativamente associado a classificação de IMC, ou seja, indivíduos obesos procuram o serviço para a redução de peso e indivíduos eutróficos para reeducação alimentar $(\mathrm{p} \leq 0,01)$. No estudo de Babinski et al. (2017), também foi observado que os principais motivos da população avaliada para a busca do acompanhamento nutricional foram redução do peso e reeducação alimentar. Contudo, não realizaram associações com o estado nutricional.

A respeito das variáveis de saúde e hábitos. No que diz respeito a função intestinal, observouse que os indivíduos avaliados possuem em grande maioria a função intestinal normal 43,9\% $(\mathrm{n}=25)$ e $35,1 \%(n=20)$ conforme as classificações 3 e 4 da Escala de Bristol respectivamente. Para função 
urinária, $66,7 \%(\mathrm{n}=22)$ e $28,1 \%(\mathrm{n}=16)$ apresentaram adequado grau de hidratação conforme a classificação 2 e 3 da escala de coloração da urina de Armstrong. Quanto a função intestinal, Santos et al. (2018), evidenciaram que, $71,9 \%$ das participantes referiram apresentar adequado funcionamento intestinal. Achados semelhantes foram encontrados por Saccon et al. (2015), que avaliou a função intestinal de 785 indivíduos, na primeira consulta nutricional, e de 351 indivíduos na última consulta. Destes, $72,1 \%(n=576)$ e $(72,1 \%)(n=253)$ relataram ter a função intestinal normal, na primeira e última consulta, respectivamente. Estes achados representam concordância com este estudo e podem estar relacionados com os hábitos alimentares, assim como com uma boa ingestão hídrica dos indivíduos.

Resultados referentes ao consumo de água, evidenciaram que 28,1\% consumiam até 1 litro, $59,7 \%$ entre 1 e 2 litros e 12,4\% consumiam mais de 2,5 litros ao dia. A maioria dos pacientes 43,9\% $(\mathrm{n}=25)$ dormia em média 8 horas por dia e $40,4 \%(\mathrm{n}=23)$ não fazia uso de nenhum medicamento. Adami et al. (2019), avaliaram a ingestão hídrica de dois grupos de mulheres pertencendo a faixa etária de 50 a 59 anos, um Grupo A (não recebiam orientação nutricional), e um Grupo B (recebiam orientação nutricional). Nas participantes do Grupo B, observaram adequado consumo de água em $61,8 \%(n=32)$, e para o Grupo A, $72,4 \%(n=42)$, das participantes, apresentaram o consumo de água abaixo do recomendado. Isto demonstra a importância do acompanhamento nutricional para obter-se uma ingestão hídrica adequada as recomendações.

Do total da amostra, $75,4 \%(n=43)$ dos pacientes praticavam atividade física, destes $24,6 \%$ $(\mathrm{n}=14)$ realizavam caminhadas, $38,6 \%(\mathrm{n}=22)$ faziam atividades com duração de 1 hora e frequência de 3 vezes na semana (36,8\%). Dammero et al. (2019), avaliaram a prática de atividade física na primeira e na última consulta em um acompanhamento nutricional de indivíduos adultos e hipertensos. Pôde ser observado que, dos indivíduos avaliados, 57,8\% e 52,6\% na primeira e última consulta, respectivamente não praticavam atividade física. Verificou-se que mesmo havendo redução da inatividade física na última consulta, a diferença entre os percentuais é reduzida, sendo um resultado divergente deste estudo. Na pesquisa de Babinsk et al. (2017), em concordância com este estudo, observaram que dentre a população avaliada, a maioria dos adultos (62\%) praticavam atividade física. Conforme Polisseni e Ribeiro (2014), atividade física foi avaliada em 280 indivíduos adultos, com idade média de 47,5 \pm 9,7 anos. Dentre os indivíduos avaliados $77 \%$ realizavam atividade física com regularidade. Quanto a modalidade escolhida, a que obteve maior prevalência foi corrida com $84,4 \%$, seguida por musculação $(77,6 \%)$ e caminhadas $(64,5 \%)$. A prática de atividade física atua como auxílio na promoção e prevenção da saúde, evitando o aparecimento das DCNTs e contribuindo para evolução na qualidade de vida da população (POLISSENI; RIBEIRO, 2014; LUCIANO et al., 2016). As associações com variáveis clínicas e de estilo de vida estão descritas na Tabela 1.

Em relação aos exames laboratoriais $82,5 \%(n=47)$ dos pacientes possuíam exames, destes $42,9 \%(\mathrm{n}=25)$ com resultados do último mês. Quanto a avaliação de resultados, apresentaram valores normais 59,6\% $(n=34)$ para glicemia em jejum $(\mathrm{GJ}), 49,1 \%(\mathrm{n}=28)$ para colesterol total $(\mathrm{CT}), 40,4 \%$ $(\mathrm{n}=23)$ para HDL, 52,6\% $(\mathrm{n}=30)$ para LDL e 59,6\% $(\mathrm{n}=34)$ para triglicerídeos $(\mathrm{TG})$. Na Tabela 2 são descritas as associações entre IMC, exames bioquímicos e parâmetros antropométricos e na Tabela 3 são descritas as associações entre sexo, exames bioquímicos e parâmetros antropométricos.

Diferente deste resultado, em uma pesquisa semelhante, com uma população adulta e idosa, ambos os sexos e idade média de 40,6 $\pm 16,0$ anos foi possível encontrar alterações para as dosagens bioquímicas citadas. As comparações foram realizadas entre sexos (masculino e feminino) e fases de vida (adultos e idosos). Quanto a CT, 32,1\% dos idosos apresentaram valores elevados, enquanto $10,8 \%$ dos adultos. Já os TG, 33,9\% idosos e 16,5\% adultos apresentaram hipertrigliceridemia. Contudo, para colesterol HDL o resultado foi semelhante a esta pesquisa, homens com níveis baixos de HDL (27,5\%) em comparação com as mulheres (14,6\%). Para glicemia, também em discordância com este estudo, a maioria dos indivíduos (23\%) apresentaram tolerância diminuída a glicose (préDM). A prevalência de tolerância diminuída foi maior entre os homens $(29,7 \%)$ em comparação com 
as mulheres $(17,7 \%)$ e nos idosos $(35,7 \%)$ quando comparado aos adultos (21\%) (LOPES et al., 2008).

Tabela 1 - Associação entre o IMC, variáveis clínicas e de estilo de vida, Santa Maria - RS, 2018

\begin{tabular}{|c|c|c|c|c|c|c|c|c|}
\hline \multirow[b]{3}{*}{ Variável } & \multirow[b]{3}{*}{ Resposta } & \multicolumn{6}{|c|}{ Classificação do IMC } & \multirow[b]{3}{*}{$\mathbf{P}$} \\
\hline & & \multicolumn{2}{|c|}{ Eutrofia } & \multicolumn{2}{|c|}{ Sobrepeso } & \multicolumn{2}{|c|}{ Obesidade } & \\
\hline & & $\mathbf{n}$ & $\%$ & $\mathbf{n}$ & $\%$ & $\mathbf{n}$ & $\%$ & \\
\hline \multirow[t]{3}{*}{ Faixa Etária } & 20 a 35 anos & 5 & $38,5 \%$ & 9 & $40,9 \%$ & 8 & $36,4 \%$ & 0,966 \\
\hline & 35 a 45 anos & 4 & $30,8 \%$ & 7 & $31,8 \%$ & 9 & $40,9 \%$ & \\
\hline & $>45$ anos & 4 & $30,8 \%$ & 6 & $27,3 \%$ & 5 & $22,7 \%$ & \\
\hline \multirow[t]{2}{*}{ Sexo } & Masculino & 6 & $46,2 \%$ & 5 & $22,7 \%$ & 8 & $36,4 \%$ & 0,395 \\
\hline & Feminino & 7 & $53,8 \%$ & 17 & $77,3 \%$ & 14 & $63,6 \%$ & \\
\hline \multirow[t]{2}{*}{ Estado civil } & Solteiro & 8 & $66,7 \%$ & 10 & $45,5 \%$ & 10 & $47,6 \%$ & 0,509 \\
\hline & Casado & 4 & $33,3 \%$ & 12 & $54,5 \%$ & 11 & $52,4 \%$ & \\
\hline Motivo do & Redução do peso & 3 & $23,1 \%$ & 13 & $59,1 \%$ & 20 & $90,9 \%$ & 0,000 \\
\hline \multirow{4}{*}{ acompanhamento } & Reeducação alimentar & 5 & $38,5 \%$ & 3 & $13,6 \%$ & 1 & $4,5 \%$ & \\
\hline & Aumento do peso & 1 & $7,7 \%$ & - & - & - & - & \\
\hline & DCNTs & 1 & $7,7 \%$ & 5 & $22,7 \%$ & 1 & $4,5 \%$ & \\
\hline & Outro & 3 & $23,1 \%$ & 1 & $4,5 \%$ & - & - & \\
\hline \multirow{7}{*}{$\begin{array}{l}\text { Patologias } \\
\text { existentes }\end{array}$} & Nenhuma & 5 & $38,5 \%$ & 8 & $36,4 \%$ & 9 & $40,9 \%$ & 0,571 \\
\hline & $\begin{array}{l}\text { Diabetes Mellitus/pré- } \\
\text { diabetes }\end{array}$ & 1 & $7,7 \%$ & 6 & $27,3 \%$ & 4 & $18,2 \%$ & \\
\hline & Hipertensão arterial & 2 & $15,4 \%$ & 2 & $9,1 \%$ & 3 & $13,6 \%$ & \\
\hline & Problemas ósseos & 1 & $7,7 \%$ & 1 & $4,5 \%$ & - & - & \\
\hline & Alergia/intolerância & 1 & $7,7 \%$ & 1 & $4,5 \%$ & 2 & $9,1 \%$ & \\
\hline & Outro & 3 & $23,1 \%$ & 4 & $18,2 \%$ & 1 & $4,5 \%$ & \\
\hline & $\mathrm{DM}+\mathrm{HAS}$ & - & - & - & - & 3 & $13,6 \%$ & \\
\hline \multirow[t]{2}{*}{ Atividade Física } & Não & 2 & $15,4 \%$ & 5 & $22,7 \%$ & 8 & $36,4 \%$ & 0,348 \\
\hline & Sim & 11 & $84,6 \%$ & 17 & $77,3 \%$ & 14 & $63,6 \%$ & \\
\hline
\end{tabular}

Notas: DCNTs = Doenças Crônicas Não Transmissíveis, HAS = Hipertensão Arterial Sistêmica, DM = Diabetes Mellitus.

Fonte: os autores.

Na pesquisa de Venturini et al. (2013), na qual analisaram indivíduos idosos de ambos os sexos e com maior proporção de idade entre 60 e 69 anos, a glicemia média não demonstrou diferença estatística entre as categorias de IMC (eutróficos 113,2 $\mathrm{mg} / \mathrm{dl} \pm 53,8$; sobrepeso 106,5 $\mathrm{mg} / \mathrm{dl} \pm 36,9$; obesos $105,1 \mathrm{mg} / \mathrm{dl} \pm 26,7$ ), assim como nesta pesquisa. Para perfil lipídico, diferente desta pesquisa, as mulheres obesas apresentaram maior frequência de HDL baixo (67\%), em comparação com os homens obesos (56\%). Quanto a ocorrência de hipertrigliceridemia, 64\% das mulheres tiveram alteração em comparação com 50\% dos homens e $70 \%$ das mulheres e $38,9 \%$ dos homens apresentaram hipercolesterolemia. Estes achados podem estar associados a presença de atividade física, na maioria dos indivíduos avaliados a prática de atividade física está presente pela faixa etária média mais jovem da amostra.

Em relação ao perfil antropométrico, o IMC médio foi de $29,4 \pm 6,1 \mathrm{~kg} / \mathrm{m}^{2}, 38,6 \%(\mathrm{n}=22)$ com sobrepeso, $38,6 \%(\mathrm{n}=22)$ com obesidade e os demais se encontravam eutróficos $(22,8 \% ; n=$ 13). Conforme CA e a RCE, 70,2\% $(n=40)$, apresentaram risco cardiovascular, respectivamente. Quanto a CB, 45,6\% $(n=26)$ apresentaram-se eutróficos e 35,1\% $(n=20)$ sobrepeso e obesidade. Na pesquisa de Priviato et al. (2015), foram avaliados indivíduos idosos, e, conforme o IMC, 53,6\% estavam com excesso de peso, 35,7\% apresentavam-se eutróficos e 10,7\% foram diagnosticados com magreza, sem diferença estatística entre os sexos. Todavia, a prevalência de excesso de peso $(60 \%)$ foi maior entre as mulheres, enquanto no grupo dos homens, houve maior prevalência de eutrofia 
(62,5\%). Quanto ao risco cardiovascular, Dantas et al. (2015) avaliou indivíduos adultos de ambos os sexos, por meio de parâmetros antropométricos incluindo perímetro da cintura e RCE. Para o perímetro da cintura foi observado maior risco cardiovascular entre as mulheres (31\%) do que entre os homens (12,6\%). Já para RCE o risco cardiovascular foi semelhante entre homens (18,6\%) e mulheres $(18,1 \%)$. Em relação a população total do estudo $(\mathrm{n}=406)$, a prevalência de risco cardiovascular segundo perímetro da cintura foi de $24,9 \%$ e $18,2 \%$ para RCE. O percentual de indivíduos que apresentaram risco cardiovascular segundo $\mathrm{CA}$ deste estudo foi superior ao encontrado por Dantas et al. (2015).

Em relação a GJ, os indivíduos eutróficos apresentaram níveis adequados, sobrepeso e obesos em maioria níveis adequados, porém, os obesos apresentam pré-diabetes. Quanto a CA e RCE, os indivíduos eutróficos não demonstraram risco cardiovascular, enquanto, àqueles com sobrepeso e obesidade apresentaram risco cardiovascular. Conforme a $\mathrm{CB}$, os indivíduos eutróficos segundo o IMC apresentaram depleção de massa muscular, enquanto aqueles que estavam com sobrepeso apresentaram eutrofia e os obesos, em consonância com o IMC, obesidade (Tabela 2).

$\mathrm{Na}$ Tabela 3 estão descritas associações entre sexo, exames bioquímicos e parâmetros antropométricos. Os homens apresentaram valores significativamente baixos de HDL. Já as mulheres apresentaram associação significativa com risco cardiovascular aumentado enquanto os homens ao baixo risco cardiovascular.

Tabela 2 - Associação entre o IMC, exames bioquímicos e parâmetros antropométricos, Santa Maria - RS, 2018

\begin{tabular}{|c|c|c|c|c|c|c|c|c|}
\hline \multirow[b]{3}{*}{ Variável } & \multirow[b]{3}{*}{ Resposta } & \multicolumn{6}{|c|}{ Classificação IMC } & \multirow{3}{*}{$\mathbf{p}$} \\
\hline & & \multicolumn{2}{|c|}{ Eutrofia } & \multicolumn{2}{|c|}{ Sobrepeso } & \multicolumn{2}{|c|}{ Obesidade } & \\
\hline & & $\mathbf{n}$ & $\%$ & n & $\%$ & $\mathbf{n}$ & $\%$ & \\
\hline \multirow[t]{3}{*}{ GJ } & Normal & 11 & $100,0 \%$ & 14 & $82,4 \%$ & 9 & $52,9 \%$ & 0,012 \\
\hline & Pré-diabetes & - & - & 1 & $5,9 \%$ & 7 & $41,2 \%$ & \\
\hline & Diabetes & - & - & 2 & $11,8 \%$ & 1 & $5,9 \%$ & \\
\hline \multirow[t]{3}{*}{$\mathrm{CT}$} & Normal & 6 & $60,0 \%$ & 10 & $58,8 \%$ & 12 & $70,6 \%$ & 0,823 \\
\hline & Limítrofe & 2 & $20,0 \%$ & 4 & $23,5 \%$ & 4 & $23,5 \%$ & \\
\hline & Elevado & 2 & $20,0 \%$ & 3 & $17,6 \%$ & 1 & $5,9 \%$ & \\
\hline \multirow[t]{2}{*}{ HDL } & Normal & 5 & $50,0 \%$ & 8 & $47,1 \%$ & 10 & $58,8 \%$ & 0,360 \\
\hline & Baixo & 1 & $10,0 \%$ & 1 & $5,9 \%$ & 4 & $23,5 \%$ & \\
\hline \multirow[t]{2}{*}{ LDL } & Normal & 6 & $60,0 \%$ & 13 & $81,3 \%$ & 11 & $64,7 \%$ & 0,713 \\
\hline & Elevado & 2 & $20,0 \%$ & 1 & $6,3 \%$ & 2 & $11,8 \%$ & \\
\hline \multirow[t]{2}{*}{ TG } & Normal & 7 & $70,0 \%$ & 13 & $81,3 \%$ & 14 & $82,4 \%$ & 0,301 \\
\hline & Elevado & 2 & $20,0 \%$ & - & - & - & - & \\
\hline \multirow[t]{2}{*}{$\mathrm{CA}$} & Sem risco cardiovascular & 8 & $66,7 \%$ & 1 & $5,3 \%$ & - & - & $\leq 0,01$ \\
\hline & Com risco cardiovascular & 4 & $33,3 \%$ & 18 & $94,7 \%$ & 18 & $100,0 \%$ & \\
\hline \multirow[t]{2}{*}{$\mathrm{RCE}$} & Sem risco cardiovascular & 7 & $58,3 \%$ & 2 & $10,5 \%$ & - & - & $\leq 0,01$ \\
\hline & Com risco cardiovascular & 5 & $41,7 \%$ & 17 & $89,5 \%$ & 18 & $100,0 \%$ & \\
\hline \multirow[t]{4}{*}{ CB } & Eutrofia & 8 & $66,7 \%$ & 14 & $73,7 \%$ & 4 & $22,2 \%$ & $\leq 0,01$ \\
\hline & Depleção & 3 & $25,0 \%$ & - & - & - & - & \\
\hline & Sobrepeso & - & - & 3 & $15,8 \%$ & 5 & $27,8 \%$ & \\
\hline & Obesidade & 1 & $8,3 \%$ & 2 & $10,5 \%$ & 9 & $50,0 \%$ & \\
\hline
\end{tabular}

Notas: $\mathrm{GJ}=$ glicemia de jejum, $\mathrm{CT}=$ colesterol total, $\mathrm{HDL}=$ lipoproteína de alta densidade, $\mathrm{LDL}=$ lipoproteína de baixa densidade, $\mathrm{TG}=$ triglicerídeos, $\mathrm{CA}=$ circunferência abdominal, $\mathrm{RCE}=$ relação cintura/estatura, $\mathrm{CB}=$ circunferência do braço.

Fonte: os autores. 
Tabela 3 - Associação entre sexo com exames bioquímicos e parâmetros antropométricos, Santa Maria - RS, 2018

\begin{tabular}{llccccc}
\hline & & \multicolumn{5}{c}{ Sexo } \\
\cline { 3 - 6 } Variável & Resposta & Masculino & Feminino & \\
\cline { 2 - 5 } GJ & Normal & 10 & $71,4 \%$ & 24 & $77,4 \%$ & 0,856 \\
& Pré-diabetes & 3 & $21,4 \%$ & 5 & $16,1 \%$ & \\
& Diabetes & 1 & $7,1 \%$ & 2 & $6,5 \%$ & \\
\hline CT & Normal & 11 & $78,6 \%$ & 17 & $56,7 \%$ & 0,453 \\
& Limítrofe & 2 & $14,3 \%$ & 8 & $26,7 \%$ & \\
& Elevado & 1 & $7,1 \%$ & 5 & $16,7 \%$ & \\
\hline HDL & Normal & 6 & $42,9 \%$ & 17 & $56,7 \%$ & 0,000 \\
& Baixo & 6 & $42,9 \%$ & - & - & \\
\hline LDL & Normal & 11 & $78,6 \%$ & 19 & $65,5 \%$ & 0,522 \\
& Elevado & 2 & $14,3 \%$ & 3 & $10,3 \%$ & \\
\hline CA & Normal & 11 & $78,6 \%$ & 23 & $79,3 \%$ & 1,000 \\
& Elevado & 1 & $7,1 \%$ & 1 & $3,4 \%$ & \\
\hline CB & Sem risco cardiovascular & 7 & $41,2 \%$ & 2 & $6,3 \%$ & 0,005 \\
& Com risco cardiovascular & 10 & $58,8 \%$ & 30 & $93,8 \%$ & \\
\hline RCE & Eutrofia & 9 & $52,9 \%$ & 17 & $53,1 \%$ & 0,343 \\
& Depleção & 2 & $11,8 \%$ & 1 & $3,1 \%$ & \\
& Sobrepeso & 1 & $5,9 \%$ & 7 & $21,9 \%$ & \\
\hline NG & Obesidade & 5 & $29,4 \%$ & 7 & $21,9 \%$ & \\
\hline Sem risco cardiovascular & 5 & $29,4 \%$ & 4 & $12,5 \%$ & 0,244 \\
& Com risco cardiovascular & 12 & $70,6 \%$ & 28 & $87,5 \%$ & \\
\hline
\end{tabular}

Notas: $\mathrm{GJ}=$ glicemia de jejum, $\mathrm{CT}=$ colesterol total, $\mathrm{HDL}=$ lipoproteína de alta densidade, $\mathrm{LDL}=$ lipoproteína de baixa densidade, $\mathrm{TG}=$ triglicerídeos, $\mathrm{CA}=$ circunferência abdominal, $\mathrm{RCE}=$ relação cintura/estatura, $\mathrm{CB}=$ circunferência do braço.

Fonte: os autores.

\section{CONCLUSÃO}

Com este estudo, pôde-se observar a relação entre parâmetros antropométricos, bioquímicos e estilo de vida. Por meio dos resultados apresentados foi possível inferir a relação entre o estado nutricional e repercussão metabólica evidenciado pelo aumento dos níveis glicêmicos pelos indivíduos com excesso de peso. Fato este, relacionado a maior incidência e aumento da morbimortalidade por DCNTs.

Devido a isto, destacamos a relevância da avaliação e do acompanhamento nutricional, assim como, promoção a práticas de educação em saúde a fim de prevenir o surgimento e as complicações associadas ao excesso de peso e demais fatores de risco para complicações metabólicas em adultos e idosos.

\section{REFERÊNCIAS}

ABESO. Associação Brasileira para o Estudo da Obesidade e da Síndrome Metabólica Diretrizes brasileiras de obesidade 2009/2010 / ABESO - Associação Brasileira para o Estudo da Obesidade e da Síndrome Metabólica. AC Farmacêutica. $3^{\text {a }}$ edição, 2009.

ADAMI, F. S.; BERTANI, J. P. B. Experiências acadêmicas de estudantes e egressos na área da nutrição - Univates. $1^{\text {a }}$ edição. Lajeado: Editora Univates, 2019. 
AMORIM, T. C.; BURGOS, M. G. P. A.; CABRAL, P. C. Perfil clínico e antropométrico de pacientes idosos com diabetes mellitus tipo 2 atendidos em ambulatório. Sciencia Médica, v. 27, $\mathrm{n}$. $3,2017$.

ARMSTRONG, L.E. et al. Urinary indices of hydration status. International Journal of Sport Nutrition, v. 4, n. 3, p. 265-279, 1994.

BABINSKI, J. M. et al. Perfil nutricional de pacientes atendidos no ambulatório de especialidades em Nutrição da URI-FW. Revista de Enfermagem FW. v. 13, n. 13, p. 41-54, 2017.

BORG, G. Escalas de Borg para a Dor e o Esforço Percebido. São Paulo: Manole, 2000.

BRASIL, Ministério do Desenvolvimento Social e Combate à Fome. Marco de referência de educação alimentar e nutricional para as políticas públicas. Brasília, Ministério da Saúde Secretaria Nacional de Segurança Alimentar e Nutricional, 2012.

BRASIL. Secretaria de Atenção à Saúde. Departamento de Atenção Básica. Estratégias para o cuidado da pessoa com doença crônica: diabetes mellitus. Cadernos de Atenção Básica $\mathrm{n}^{\circ} 36$. Brasília, DF. Ministério da Saúde, 2013.

BRASIL, Ministério da Saúde. Secretaria de Atenção à Saúde. Departamento de Atenção Básica. Guia alimentar para a População Brasileira. 2ª edição, Brasília: Ministério da Saúde, 2014.

BRASIL. Secretaria de Vigilância em Saúde. Departamento de Vigilância de Doenças e Agravos não Transmissíveis e Promoção da Saúde. Vigitel Brasil 2014: vigilância de fatores de risco e proteção para doenças crônicas por inquérito telefônico. Brasília: Ministério da Saúde. p.152, 2015.

BRASIL. Diretrizes da Sociedade Brasileira de Diabetes 2017-2018. Editora Clannad. São Paulo, 2017.

BRASIL. Sociedade Brasileira de Cardiologia. Atualização da Diretriz Brasileira de Dislipidemias e prevenção da Aterosclerose - 2017, v. 109, n. 1, 2017.

BRASIL. Secretaria de Vigilância em Saúde. Departamento de Vigilância de Doenças e Agravos não Transmissíveis e Promoção da Saúde. Saúde Brasil 2017: uma análise da situação de saúde e os desafios para o alcance dos objetivos de desenvolvimento sustentável recurso eletrônico. Brasília: Ministério da Saúde, 2018.

BRASIL. Secretaria de Vigilância em Saúde. Departamento de Vigilância de Doenças e Agravos não Transmissíveis e Promoção da Saúde. Relatório do III Fórum de Monitoramento do Plano de Ações Estratégicas para o Enfrentamento das Doenças Crônicas não Transmissíveis no Brasil - recurso eletrônico. Brasília: Ministério da Saúde, 2018.

BRASIL, Departamento de Vigilância de Doenças e Agravos não Transmissíveis e Promoção da Saúde. Saúde Brasil 2018 uma análise de situação de saúde e das doenças e agravos crônicos: desafios e perspectivas. Brasília: Ministério da Saúde - Secretaria de Vigilância em Saúde, 2019.

CARLUCCI, E. M. S. et al. Obesidade e sedentarismo: fatores de risco para doença cardiovascular, Comunicação em Ciências Saúde. v. 24, n. 4, p. 375-384, outubro/dezembro, 2013. 
CARVALHO, J. L. et al. Perfil de pacientes atendidos em laboratório de práticas em nutrição clínica na região central do RS. Disciplinarum Scientia. Série: Ciências da Saúde, v. 16, n. 1, p. 137-145, 2015.

CERVATO-MANCUSO, A. M.; VINCHA, K. R. R.; SANTIAGO, D. A. Educação Alimentar e Nutricional como prática de intervenção: reflexão e possibilidades de fortalecimento. Physis: Revista de Saúde Coletiva, v. 26, n. 1, p. 225-249, 2016.

CUPPARI, L. Nutrição: nas doenças crônicas não-transmissíveis. 3. ed. Barueri: Editora Manole, 2009.

DAMMERO, D. R. et al. Perfil e estado nutricional de pacientes hipertensos atendidos em um ambulatório de nutrição do sul do Brasil. Revista Brasileira de Obesidade, Nutrição e Emagrecimento, v. 13, n. 77, p. 54-60, 2019.

DANTAS, E. M. S. et al. Concordância na avaliação de risco cardiovascular a partir de parâmetros antropométricos. Revista Einstein, v. 13, n. 3, p. 376-80, 2015.

FONTES, A. M. G. G. et al. Waist-Stature Ratio And Its Relationship With Autonomic Recovery From Aerobic Exercise In Healthy Men. Scientific Reports, v. 8, n. 1, 2018.

FRANGELLA, V. S.; TCHAKMAKIAN, L. A.; PEREIRA, M. A. G. Aspectos Nutricionais e Técnicos na Área Clínica, In: SILVA, S. M. C.; MURA, J. D. P. Tratado de alimentação, nutrição e dietoterapia. São Paulo: Roca, cap. 26, p. 437-445, 2007.

IBGE. Pesquisa Nacional Por Amostras de Domicílios Contínua (PNAD Contínua) 2018. Instituto Brasileiro de Geografia e Estatística (IBGE), 2018.

IBGE. Pesquisa de orçamentos familiares 2017-2018: avaliação nutricional da disponibilidade domiciliar de alimentos no Brasil. Coordenação de Trabalho e Rendimento. Instituto Brasileiro de Geografia e Estatística (IBGE), 2020.

LOPES, A. C. S. Estado nutricional: antropometria, consumo alimentar e dosagens bioquímicas de adultos e idosos - Projeto Bambuí um estudo de base populacional. REME - Revista Mineira de Enfermagem, v. 12, n. 4, p. 483-493, 2008.

LUCIANO, A. P. et al. Nível de atividade física em adolescentes saudáveis. Revista Brasileira de Medicina do Esporte. v. 22, n. 3, p. 191-194, 2016.

MALTA, D. C. et al. A vigilância e o monitoramento das principais doenças crônicas não transmissíveis no Brasil - Pesquisa Nacional de Saúde, 2013. Revista Brasileira de Epidemiologia. v. 18, n. 2 , p. 3-16, 2015.

MOREIRA, P. V. L. et al. Comparing different policy scenarios to reduce the consumption of ultraprocessed foods in UK: impact on cardiovascular disease mortality using a modelling approach. Plos One. v. 10, n. 2, e0118353, 2015.

MOREIRAS, G. V. Nutritional issues in Spanish women; findings of the ANIBES Study. Nutrición Hospitalaria, v. 32, n. 1, p. 14-19, 2015.

MUSSOI, T. D. Avaliação nutricional na prática clínica: da gestação ao envelhecimento. 1. ed. Rio de Janeiro: Guanabara Koogan, 2014. 
ORGANIZAÇÃO PAN-AMERICANA DA SAÚDE. XXXVI Reunión del Comitê Asesor de Ivestigaciones en Salud - Encuestra Multicêntrica - Salud Beinestar y Envejecimeiento (SABE) en América Latina en el Caribe - Informe preliminar. OPAS, 2002.

POLISSENI, M. L. C.; RIBEIRO, L. C. Exercício físico como fator de proteção para a saúde em servidores públicos. Revista Brasileira de Medicina do Esporte, v. 20, n. 5, p. 340-344, 2014.

PRIVIATO, H. D. R. A. et al. Perfil clínico-nutricional e consumo alimentar de idosos do Programa Terceira Idade, Ouro Preto-MG. Demetra, v. 10, n. 2, p. 375-387, 2015.

REIS, G. M. S.; ALMEIDA, A. F. Parâmetros clínicos e nutricionais dos pacientes atendidos em um ambulatório de nutrição. Journal Braspen, v. 33, n. 2, p. 199-205, 2018.

SARAIVA, D. I.; ZEMOLIN, G. P.; ZANARDO, V. P. S. Perfil nutricional de pacientes atendidos em um ambulatório e especialidades em nutrição. Vivências: Revista Eletrônica de Extensão da URI, v. 10, n. 19, p. 113-121, 2014.

SACCON, T. D. et al. Perfil e evolução do estado nutricional de pacientes que frequentam um ambulatório de nutrição do Sul do Brasil. Nutrición clínica e dietética hospitalaria, v. 35, n. 3, p. 74-82, 2015.

SANTOS, L. G. et al. Perfil, estado nutricional e variação de peso de mulheres adultas atendidas em um ambulatório de nutrição. Nutrición clínica e dietética hospitalaria, v. 38, n. 4, p. 33-38, 2018.

SILVA, M. B. G. et al. Perfil clínico e nutricional dos indivíduos atendidos em um ambulatório de nutrição do hospital universitário (HUPAA/UFAL). Gep News, v. 1, n. 1, p. 139-144, 2018.

STEWART, A. et al. Padrões Internacionais para Avaliação Antropométrica (2011). 1. ed. Murcia: Sociedade Internacional para o Avanço da Cineantropometria (ISAK), 2001. Revisado em 2006 e 2011.

STOPA, S. R. et al. Prevalência da hipertensão arterial, do diabetes mellitus e da adesão às medidas comportamentais no Município de São Paulo, Brasil, 2003-2015. Cadernos de Saúde Pública, v. 34, n. 10, e00198717, 2018.

VENTURINI, C. D. et al. Prevalência de obesidade associada à ingestão calórica, glicemia e perfil lipídico em uma amostra populacional de idosos do Sul do Brasil. Revista Brasileira de Geriatria e Gerontologia do Rio de Janeiro, v. 16, n. 3, p. 591-601, 2013.

VIGITEL. Secretaria de Vigilância em Saúde. Departamento de Vigilância de Doenças e Agravos não Transmissíveis e Promoção da Saúde. Vigitel Brasil 2017: vigilância de fatores de risco e proteção para doenças crônicas por inquérito telefônico: estimativas sobre frequência e distribuição sociodemográfica de fatores de risco e proteção para doenças crônicas nas capitais dos 26 estados brasileiros e no Distrito Federal em 2017 - Brasília. Brasília: Ministério da Saúde, 2018 .

WORLD HEALTH ORGANIZATION. Obesity: preventing and managing the global epidemic of obesity. Report of the WHO Consultation of Obesity. Geneva: World Health Organization, 1997.

WORLD HEALTH ORGANIZATION. Global recommendations on physical activity for health. Geneva: World Health Organization, 2010. 